\title{
Achados de imagem e alternativas terapêuticas das malformações vasculares periféricas*
}

\author{
Imaging findings and therapeutic alternatives for peripheral vascular malformations
}

\author{
Lucas Moretti Monsignore ${ }^{1}$, Guilherme Seizem Nakiri ${ }^{1}$, Daniela dos Santos ${ }^{1}$, Thiago
}

Giansante Abud $^{2}$, Daniel Giansante Abud ${ }^{3}$

Resumo As malformações vasculares periféricas compreendem um espectro de lesões que se tornam aparentes no decorrer da vida e podem ser encontradas em praticamente todo o corpo. São pouco comuns e frequentemente confundidas com o hemangioma infantil. Estas doenças são completamente distintas tanto em relação à história clínica como ao prognóstico e às formas de tratamento. Nestas lesões, a história evolutiva e as características do exame físico são de extrema importância para o adequado diagnóstico clinicorradiológico, que guiará a melhor alternativa terapêutica. As classificações mais recentes dividem as malformações vasculares periféricas levando em consideração o fluxo sanguíneo (alto e baixo) e os componentes vasculares envolvidos (arteriais, capilares, linfáticos e venosos). As malformações vasculares periféricas representam um desafio diagnóstico e terapêutico, e exames complementares como tomografia computadorizada, ultrassonografia com Doppler e ressonância magnética, em conjunto com a história clínica, podem trazer informações quanto às características de fluxo e à extensão das lesões. Arteriografia e flebografia confirmam o diagnóstico, avaliam a sua extensão e orientam a decisão terapêutica. Malformações de baixo fluxo geralmente são tratadas por abordagem percutânea e injeção de agente esclerosante, enquanto para as malformações de alto fluxo o acesso é endovascular com uso de agentes embolizantes permanentes líquidos ou sólidos.

Unitermos: Malformações vasculares; Embolização terapêutica; Escleroterapia; Radiologia intervencionista.

Abstract Peripheral vascular malformations represent a spectrum of lesions that appear through the lifetime and can be found in the whole body. Such lesions are uncommon and are frequently confounded with infantile hemangioma, a common benign neoplastic lesion. In the presence of such lesions, the correlation between the clinical and radiological findings is extremely important to achieve a correct diagnosis, which will guide the best therapeutic approach. The most recent classifications for peripheral vascular malformations are based on the blood flow (low or high) and on the main vascular components (arterial, capillary, lymphatic or venous). Peripheral vascular malformations represent a diagnostic and therapeutic challenge, and complementary methods such as computed tomography, Doppler ultrasonography and magnetic resonance imaging, in association with clinical findings can provide information regarding blood flow characteristics and lesions extent. Arteriography and venography confirm the diagnosis, evaluate the lesions extent and guide the therapeutic decision making. Generally, low flow vascular malformations are percutaneously treated with sclerosing agents injection, while in high flow lesions the approach is endovascular, with permanent liquid or solid embolization agents.

Keywords: Vascular malformations; Therapeutic embolization; Sclerotherapy; Interventional radiology.

Monsignore LM, Nakiri GS, Santos D, Abud TG, Abud DG. Achados de imagem e alternativas terapêuticas das malformações vasculares periféricas. Radiol Bras. 2010;43(3):185-194.

\footnotetext{
* Trabalho realizado no Centro de Ciências das Imagens Física Médica do Hospital das Clínicas da Faculdade de Medicina de Ribeirão Preto da Universidade de São Paulo (CCIFM/HC FMRPUSP), Ribeirão Preto, SP, Brasil.

1. Médicos Residentes em Neurorradiologia Terapêutica e Radiologia Intervencionista do Hospital das Clínicas da Faculdade de Medicina de Ribeirão Preto da Universidade de São Paulo (HC-FMRPUSP), Ribeirão Preto, SP, Brasil.

2. Mestre, Radiologista Intervencionista da Clínica Documenta de Ribeirão Preto e do Hospital das Clínicas da Faculdade de Medicina de Ribeirão Preto da Universidade de São Paulo (HCFMRPUSP), Ribeirão Preto, SP, Brasil.

3. Professor Doutor, Docente Responsável pelo Serviço de Neurorradiologia Terapêutica e Radiologia Intervencionista do Hospital das Clínicas da Faculdade de Medicina de Ribeirão Preto da Universidade de São Paulo (HC-FMRPUSP), Ribeirão Preto, SP, Brasil.

Endereço para correspondência: Dr. Lucas Moretti Monsignore. CCIFM/HC-FMRPUSP. Avenida Bandeirantes, 3900, Monte
}

\section{INTRODUÇÃO}

As malformações vasculares periféricas (MVPs) são caracterizadas pelo desenvolvimento anormal de estruturas vasculares (arteriais, capilares, venosas e linfáticas) em diferentes proporções. De etiologia congênita, podem apresentar-se no decorrer da vida e em cerca de $90 \%$ dos casos já

Alegre. Ribeirão Preto, SP, Brasil, 14048-900. E-mail: lucasmonsignore@gmail.com

Recebido para publicação em 2/3/2010. Aceito, após revisão, em 23/4/2010. são visíveis ao nascimento ${ }^{(\mathbf{1})}$. A história clínica e o exame físico são diagnósticos na maioria dos casos, e exames de imagem, como a ultrassonografia com Doppler, a tomografia computadorizada e a ressonância magnética, auxiliam em casos duvidosos e são importantes para o adequado planejamento terapêutico.

A correta classificação das MVPs, com uso das terminologias atuais, guia o tratamento e evita confusões semânticas, resultando em indicações terapêuticas equivocadas. Alguns tipos de MVPs são erronea- 
mente denominadas "hemangiomas" e frequentemente confundidos com o hemangioma infantil, neoplasia benigna, geralmente autolimitada e com regressão espontânea na maioria dos casos, caracteristicamente encontrada em neonatos.

O tratamento das MVPs com auxílio dos diferentes métodos de imagem esteve em destaque nos últimos anos, principalmente graças aos ótimos resultados finais, baixos riscos para o paciente e progresso nos materiais e técnicas utilizados. Em algumas situações, pode-se associar o tratamento endovascular ao cirúrgico.

\section{CLASSIFICAÇÃO}

As MVPs e os tumores vasculares são doenças completamente distintas, tanto do ponto de vista diagnóstico, como terapêutico e das formas e da necessidade de acompanhamento, porém, juntas, podem ser denominadas anomalias vasculares. Um dos motivos para a confusão é a nomenclatura utilizada para as anomalias vasculares pelos diferentes profissionais envolvidos no tratamento dos pacientes ${ }^{(2)}$.

Radiologistas, patologistas, pediatras, dermatologistas, cirurgiões vasculares, plásticos e de cabeça e pescoço, otorrinolaringologistas e oftalmologistas são alguns dos especialistas envolvidos no primeiro atendimento, diagnóstico, tratamento e acompanhamento desses pacientes, em geral crianças, adolescentes e adultos jovens. Nomenclaturas dúbias para doenças diferentes com frequência levam a iatrogenias terapêuticas, algumas vezes com resultados catastróficos para o paciente ${ }^{(3)}$. Tratar um hemangioma infantil (tumor benigno da infância com involução espontânea na maioria dos casos) como uma malformação venosa de baixo fluxo (muitas vezes denominada "hemangioma cavernoso") pode levar a uma mutilação desnecessária. E o contrário, tratar uma malformação venosa como um hemangioma infantil também leva a insucesso terapêutico, com dispêndios desnecessários, sem a resolução do problema do paciente.

Para tentar solucionar o problema, em 1992, após anos de encontros internacionais para discutir o assunto, foi criada a Sociedade Internacional para o Estudo das Anomalias Vasculares (International So- ciety for the Study of Vascular Anomalies - ISSVA). Em 1996, durante um de seus encontros, foi criada a classificação atual das doenças (Tabela 1).

Esta classificação divide as anomalias vasculares em tumores vasculares (que incluem os hemangiomas infantis e outros tumores vasculares raros que podem ocorrer tanto em crianças como em adultos) e malformações vasculares (subclassificadas de acordo com as características do fluxo sanguíneo - alto e baixo fluxo - e de acordo com os componentes histológicos que compõem a lesão - arterial, capilar, venoso e linfático). A classificação baseou-se em achados patológicos do artigo de Mulliken e Glowacki, publicado em $1982^{(4)}$. Tumores vasculares diferenciam-se das malformações vasculares baseados em sua aparência clínica, radiológica, achados histopatológicos e comportamento biológico. $\mathrm{O}$ sufixo "oma" significa proliferação tumoral, por isso termos como "angioma", "hemangioma" e "linfangioma" são errôneos quando atribuídos a malformações vasculares e foram abolidos ${ }^{(3)}$.

As anomalias vasculares são classificadas de modo não invasivo baseando-se nos achados clínicos, na história evolutiva e nas características encontradas nos diferentes exames de imagem ${ }^{(\mathbf{5 , 6})}$ : ultrassonografia com técnica Doppler colorida e espectral, radiografia simples, angiotomografia computadorizada, angiorressonância magnéti$\mathrm{ca}^{(7)}$, flebografia e angiografia por cateter, cada um com uma importância diferente nos diversos subtipos da doença (Tabela 2).

\section{QUADRO CLÍNICO E ACHADOS NOS DIFERENTES MÉTODOS DE IMAGEM}

O quadro clínico é extremamente variável. As MVPs podem apresentar-se como: assintomáticas; pequenas máculas ao nascimento; lesões expansivas com coloração variável, associadas ou não a dor e limitação funcional; extensas deformidades com impacto estético; e insuficiência cardíaca de alto débito. Podem ser encontradas isoladamente ou como parte de uma síndrome genética $^{(8)}$ e distribuem-se por todo o corpo. Não há predomínio entre os sexos.

Estão sempre presentes ao nascimento, mesmo que nem sempre visíveis. Têm crescimento gradual, que acontece em diferen-

Tabela 1 Classificação das anomalias vasculares considerando seus componentes vasculares (ISSVA / Mulliken, 1996). (Adaptado de Enjolras et al. ${ }^{(3)}$ ).

\begin{tabular}{|c|c|}
\hline Tumores vasculares & Malformações vasculares \\
\hline $\begin{array}{l}\text { Hemangioma infantil } \\
\text { Hemangioma congênito (rapidamente in- } \\
\text { volutivo e não involutivo) } \\
\text { Angioma em tufos } \\
\text { Hemangioendotelioma kaposiforme } \\
\text { Hemangiopericitoma } \\
\text { Granuloma piogênico } \\
\text { Hemangioendotelioma de células fusifor- } \\
\text { mes } \\
\text { Outros }\end{array}$ & $\begin{array}{l}\text { Malformações vasculares de baixo fluxo } \\
\text { Malformação capilar (MC) } \\
\text { Mancha em vinho-do-Porto } \\
\text { Teleangiectasia } \\
\text { Angioqueratoma } \\
\text { Malformação venosa (MV) } \\
\text { MV comum esporádica } \\
\text { Síndrome de Bean } \\
\text { MV familiar cutânea e mucosa } \\
\text { Malformação glomuvenosa (“glomangioma”) } \\
\text { Síndrome de Maffucci } \\
\text { Malformação linfática (ML) } \\
\text { Malformações vasculares de alto fluxo } \\
\text { Malformação arterial (MA) } \\
\text { Fístula arteriovenosa (FAV) } \\
\text { Malformação arteriovenosa (MAV) } \\
\text { Malformações vasculares complexas-combinadas } \\
\text { Malformação capilar-venosa } \\
\text { Malformação capilar-linfático-venosa } \\
\text { Malformação linfático-venosa } \\
\text { Malformação capilar-artério-venosa } \\
\text { Malformação capilar-linfático-artério-venosa }\end{array}$ \\
\hline
\end{tabular}


Tabela 2 Importância dos diferentes exames de imagem em algumas anomalias vasculares. (Adaptado de Enjolras et al. ${ }^{(3)}$ ).

\begin{tabular}{lccccc}
\hline & $\begin{array}{c}\text { Hemangioma } \\
\text { infantil }\end{array}$ & MC & MV & ML & MAV \\
\hline US-Doppler & +++ & ++ & ++ & ++ & +++ \\
Radiografia & - & - & ++ & $+/-$ & + \\
Angio-TC & + & - & (flebólito/osso) & (osso) & (osso) \\
Angio-RM & ++ & - & +++ & +++ & ++ \\
Angiografia por cateter & - & - & + & - & ++ \\
\hline
\end{tabular}

MC, malformação capilar; MV, malformação venosa; ML, malformação linfática; MAV, malformação arteriovenosa. tes fases da vida dependendo dos componentes da lesão. Nunca apresentam involução espontânea (característica presente na maioria dos hemangiomas infantis).

\section{Malformações capilares (MCs)}

São as malformações vasculares mais as teleangiectasias e as manchas em vinhodo-Porto ${ }^{(9)}$. As MCs são lesões de baixo fluxo usualmente visíveis ao nascimento e apresentam-se como máculas avermelhadas de dimensões variáveis (pequenas máculas até grandes lesões que podem recobrir todo um membro) ${ }^{(\mathbf{1 0})}$. São mais comuns na cabeça e pescoço. Em geral não apresentam alteração de coloração no decorrer da vida e podem ser confundidas com precursores de malformações venosas, sendo por vezes necessária uma reavaliação da lesão meses após o nascimento para confirmar o diagnóstico. liação destas lesões quando há a suspeita de outra lesão vascular, geralmente profunda, mimetizando uma MC, é a ultrassonografia com técnica Doppler. No caso de uma MC, a ultrassonografia com Doppler não apresentará achado específico, enquanto no caso de outra lesão vascular profunda, mimetizando uma MC, os achados serão específicos da doença ${ }^{(\mathbf{6})}$.

\section{Malformações venosas (MVs)}

As MVs já estão presentes ao nascimento e podem desenvolver-se em qualquer período da infância e adolescência. Usualmente apresentam-se como massas azuladas, compressíveis, às vezes associadas a pequenas nodulações endurecidas, que correspondem a flebólitos. Não há alteração da temperatura da lesão ou presença de frêfrequentemente diagnosticadas e incluem

O método de imagem indicado para ava- mito à palpação. Podem apresentar variação de suas dimensões, dependendo da posição ou da manobra de Valsalva ${ }^{(2)}$.

A ressonância magnética é o método de imagem que fornece mais informações sobre a real extensão da lesão e sua relação com estruturas adjacentes (Figuras 1 e 2). São iso- ou hipointensas nas sequências ponderadas em $\mathrm{T} 1$, podendo ser discretamente hiperintensas caso contenham gordura em seu interior. Pequenas áreas de ausência de sinal em todas as sequências representam calcificações distróficas ou flebólitos. Classicamente, apresentam hiperintensidade nas sequências ponderadas em T2, sequência que é mais sensível para demonstrar a real extensão da lesão. Após a administração de contraste, haverá realce homogêneo ou heterogêneo da lesão, permitindo diferenciação exata da lesão em relação ao tecido perilesional. É o método de escolha para acompanhar os resultados após escleroterapia $^{(11)}$ (Figura 2). Estas áreas mostram-se heterogêneas tanto em T1 quanto em T2, e um menor realce em relação às áreas da lesão não tratadas ${ }^{(7)}$.

A avaliação com Doppler colorido e espectral demonstra fluxo monofásico ou bifásico em cerca de $80 \%$ das lesões. A arteriografia não tem papel importante na avaliação das MVs, uma vez que a grande maioria não apresentará achados específicos ou apenas um discreto blush venoso ou capilar tardio. As artérias que suprem a malformação podem ser normais ou discretamente ectasiadas, e as veias de drenagem podem apresentar-se dilatadas.

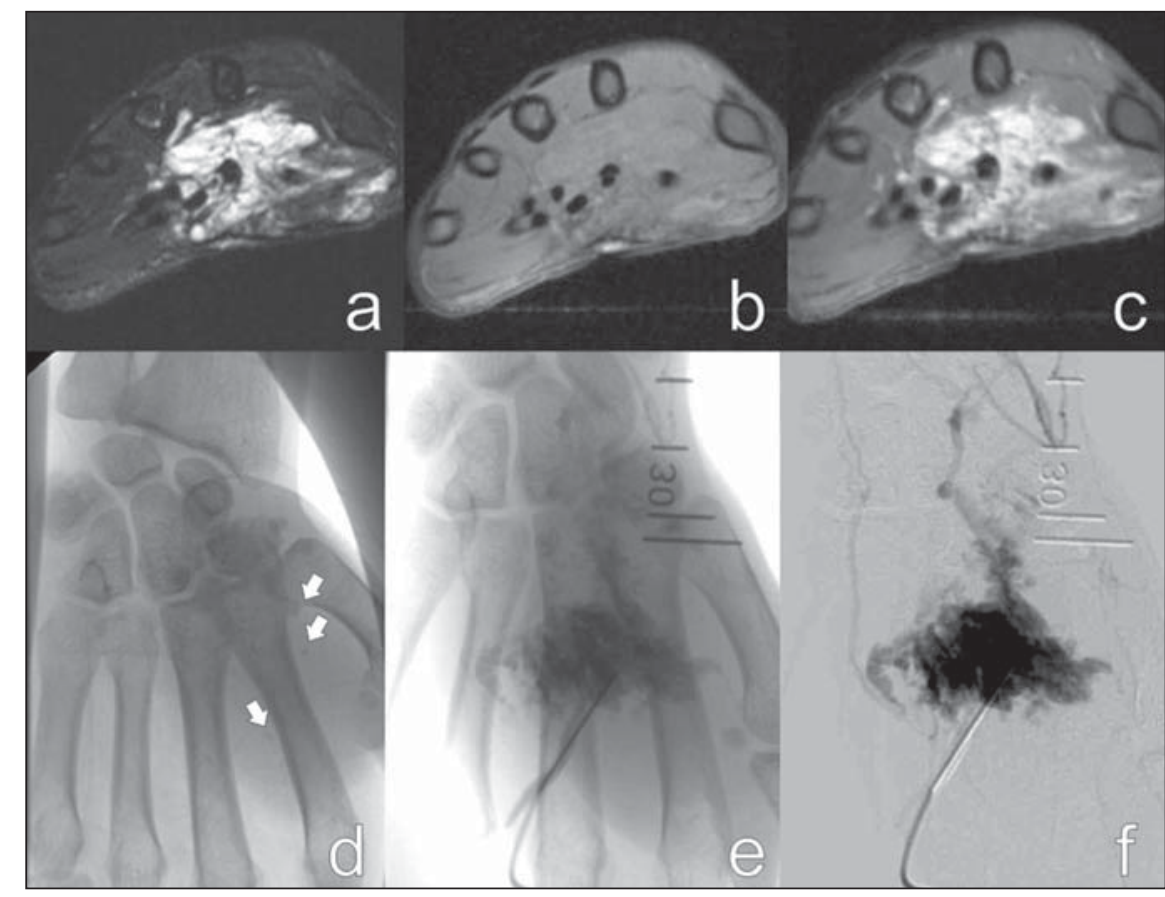

Figura 1. Malformação venosa. Sexo masculino, 36 anos, lesão arroxeada e compressível na face palmar da mão esquerda, presente desde o nascimento e com crescimento na adolescência. Ressonância magnética, sequências ponderadas em (a) T2 com saturação de gordura, (b) T1 com saturação de gordura e (c) T1 com saturação de gordura após administração de contraste, evidencia lesão de limites precisos com hipersinal em T2, isossinal à musculatura em T1 e realce homogêneo após a administração de contraste na face palmar da mão esquerda, envolvendo os tendões flexores dos dedos. Radiografia simples (d) demonstra pequenos flebólitos na topografia da lesão (setas). Flebografia (e,f) por punção direita precedendo a escleroterapia com álcool absoluto evidencia lesão dismórfica, com retorno venoso em sentido habitual. 


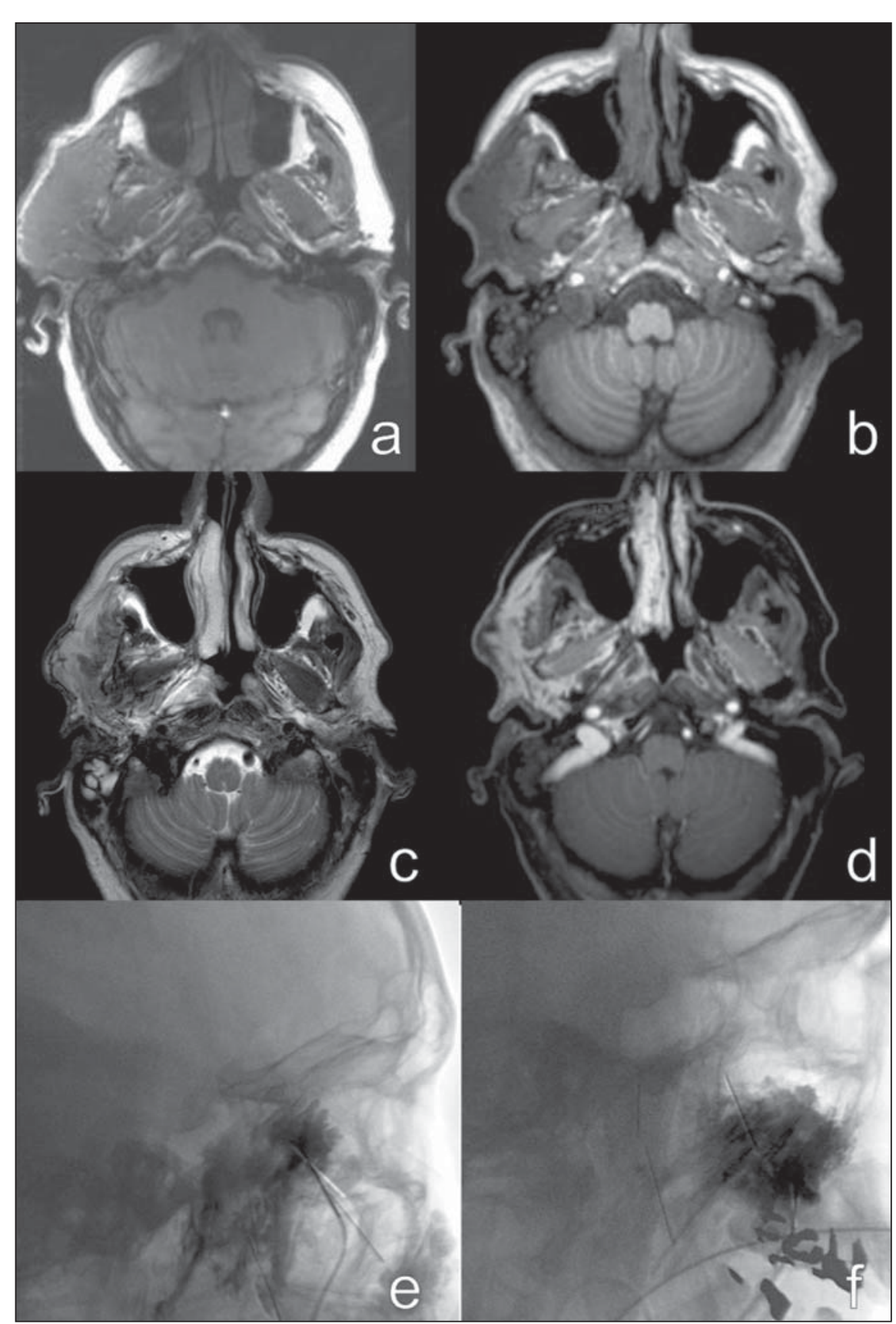

Figura 2. Malformação venosa. Sexo masculino, 55 anos, deformidade facial associado a lesão cutânea arroxeada, presente desde o nascimento. Ressonância magnética, (a) sequência ponderada em T1, realizada no início do tratamento percutâneo, evidencia assimetria facial, maior à direita, à custa de formação expansiva de limites precisos e irregulares, abrangendo o subcutâneo e a musculatura da região malar, com isossinal à musculatura em T1. Ressonância magnética, sequências ponderadas em (b) T1, (c) T2 e (d) T1 com saturação de gordura após administração de contraste, realizada quatro anos após o início do tratamento, evidencia melhora na assimetria facial, com redução da lesão previamente descrita, que demonstra hipersinal em T2 e realce homogêneo após a administração de contraste. Flebografia (e,f) por punção direita precedendo uma das sessões de escleroterapia com álcool absoluto evidencia lesão multicavitada em hemiface direita.

A ultrassonografia é o primeiro método utilizado para avaliação das MVs, por não ser invasivo e ser amplamente disponível. Caracterizam-se por massas heterogêneas, predominantemente hipoecogênicas, apre- sentando em seu interior estruturas tubulares hipoecoicas, que representam os canais vasculares da lesão. Quando detectados, os flebólitos revelam-se como estruturas hiperecogênicas com sombra acústica posterior.
A tomografia computadorizada, mesmo com administração de contraste, fornece informações limitadas em relação à lesão, habitualmente subestimando suas dimensões, além de expor o paciente à radiação ionizante. Neste método, assim como na radiografia convencional, um achado frequente é a presença de flebólitos (Figura 1) e o espessamento da cortical óssea, quando há intima relação entre a lesão e o osso.

A flebografia por punção direta é considerada padrão ouro e indicada nas situações em que se necessita confirmação do diagnóstico ou planejamento terapêutico. Foram descritos padrões flebográficos distintos considerando a morfologia (espongiforme, cavitário e dismórfico) e o retorno venoso (tipo I: isolado da drenagem venosa normal; tipo II: drenagem venosa em sentido habitual, com válvulas competentes; tipo III: veias de drenagem ectasiadas e apresentando válvulas incompetentes; tipo IV: completamente composta de veias ectasiadas e displásicas) ${ }^{(2)}$. Esta classificação ajuda no planejamento terapêutico e sugere o prognóstico. Lesões cavitárias e dismórficas são tecnicamente mais simples de serem tratadas, apesar das lesões dismórficas apresentarem maiores taxas de recorrência. Lesões espongiformes necessitam múltiplas sessões de escleroterapia e múltiplas punções por sessão.

\section{Malformações linfáticas (MLs)}

Cerca de $80 \%$ das MLs são visíveis antes de um ano de vida. São encontradas predominantemente na região da cabeça, pescoço e axilas (95\%) e apresentam-se como massas sem alteração da coloração da pele, quando superficiais, e não compressíveis às manobras (em contraste com as MVs). Podem ser classificadas em: macrocísticas, quando apresentam áreas císticas de dimensões variáveis; microcísticas, quando apresentam áreas menores que $2 \mathrm{~mm}$ em uma matriz sólida; ou mistas, quando apresentam achados dos dois subtipos anteriores ${ }^{(\mathbf{6})}$. São lesões que podem ter dimensões que variam de acordo com processos inflamatórios regionais ou sistêmicos. A ressonância magnética é o melhor exame de imagem para determinar a extensão da lesão e controlar a terapêutica (Figura 3). Caracterizam-se por massas lobuladas, com iso- ou hipossinal nas sequências pon- 


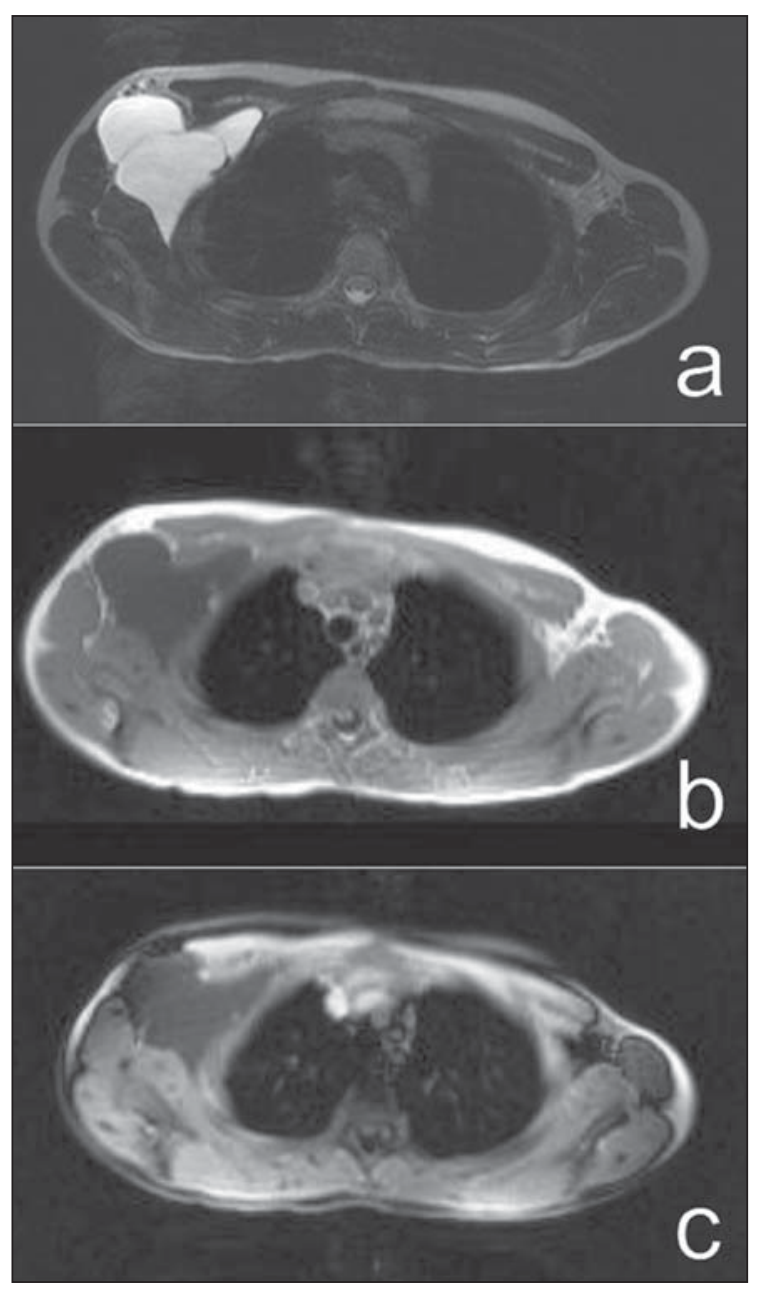

postas por tecido malformativo denominado nicho ou nidus. Apresentam-se como massa coberta por pele de coloração normal ou angiomatosa, geralmente lisa e brilhante ${ }^{(\mathbf{1 3})}$, com aumento da temperatura local, e podem apresentar frêmito e sopro. As veias de drenagem podem tornar-se evidentes e tortuosas, e traumas locais podem levar a sangramentos de grande monta com risco à vida. A ultrassonografia é método de avaliação inicial e demonstra estruturas tubulares anecoicas, sem massa de partes moles bem definida. Ao estudo Doppler, a lesão apresenta áreas de shunt arteriovenoso, com altas velocidades de pico sistólico e veias ectasiadas com fluxo arterializado. A ressonância magnética (Figura 5) evidencia diversas áreas de ausência de sinal (flow-void) nas sequências ponderadas em $\mathrm{T} 1$ e $\mathrm{T} 2$, que correspondem às artérias nutridoras e ao nidus malformativo.

Figura 3. Malformação linfática, tipo macrocística. Sexo feminino, 6 anos, com abaulamento indolor e sem lesão cutânea na região axilar direita observada aos seis meses de idade. Ressonância magnética, sequências ponderadas em (a) T2, (b) T1 e (c) T1 com saturação de gordura após administração de contraste, evidencia lesão de limites precisos com alguns finos septos em seu interior situada na região axilar direita, apresentando hipersinal em T2, hipossinal em T1 e discreto realce de alguns septos após a administração do contraste. Geralmente não são observadas massas junto dos vasos patológicos, o que facilita a diferenciação das MAVs em relação a tumores hipervasculares ${ }^{(7)}$. Paralelamente, na tomografia computadorizada mantém-se o aspecto de múltiplas artérias nutridoras ectasiadas, com contrastação precoce das veias de drenagem, sem massa significativa interponente. A arteriografia (Figuras 5 e 6) é diagnóstica e padrão ouro para o

deradas em T1 e hipersinal nas sequências ponderadas em T2. Após a administração de contraste, pode haver discreto halo de realce ou realce nos septos nas lesões macrocísticas e ausência ou mínimo realce nas lesões microcísticas. Pode ser observado linfedema perilesional ${ }^{(7)}$. A ultrassonografia, assim como para outras malformações vasculares, é método utilizado para avaliação inicial. O subtipo macrocístico é caracterizado por massa cística multiloculada, sem fluxo ao estudo Doppler, enquanto o subtipo microcístico apresenta-se hiperecogênico $^{(\mathbf{1 2})}$. A tomografia computadorizada demonstra massas com baixa atenuação, ocasionalmente apresentando níveis líquidos, com mínimo realce septal e periférico. A angiografia não tem valor diagnóstico neste tipo de malformação vascular (Figura 4).

\section{Malformações arteriovenosas (MAVs)}

As MAVs caracterizam-se por comunicação anormal entre artérias e veias, inter-

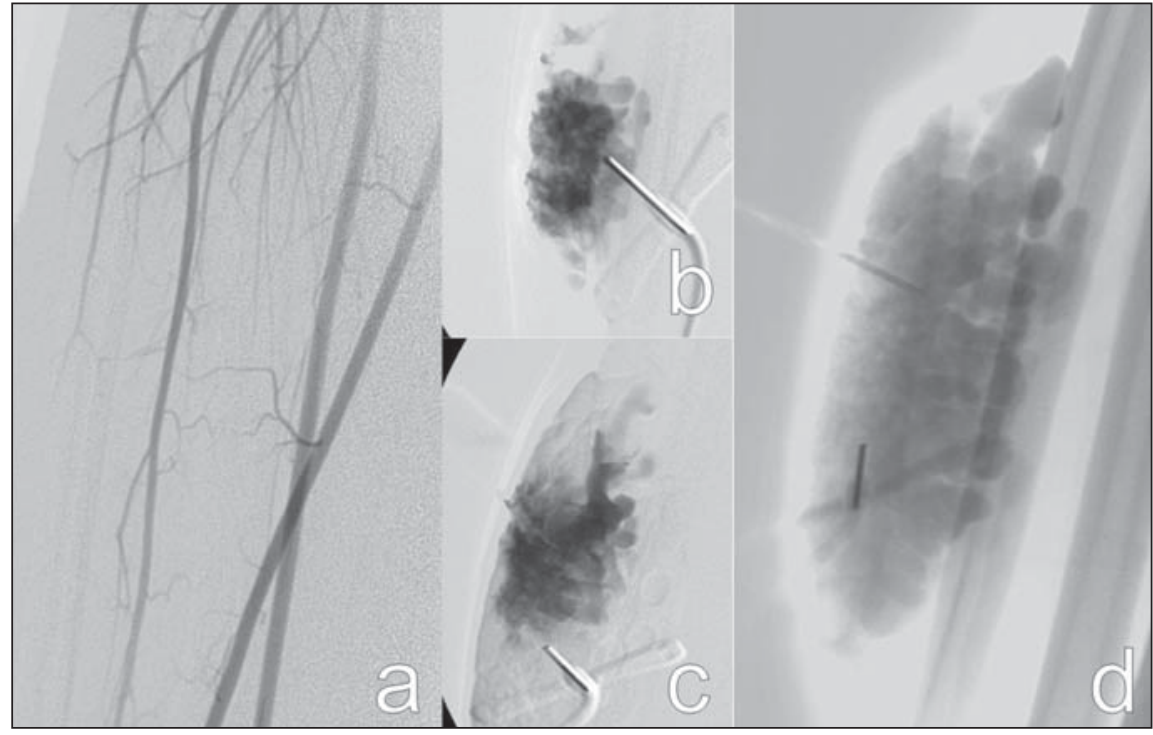

Figura 4. Malformação linfática, tipo macrocística. Sexo feminino, 25 anos, com abaulamento indolor no antebraço direito e sem lesão cutânea associada desde a infância. (a) Angiografia seletiva por cateter do membro superior direito não evidenciou lesões. (b,c) Punções percutâneas com injeção de solução iodada após aspiração de conteúdo citrino precedendo a escleroterapia demonstra múltiplas cavidades de tamanhos variados que se comunicam. (d) Aspecto fluoroscópico imediatamente após aplicação percutânea de solução de álcool absoluto e contraste iodado. 


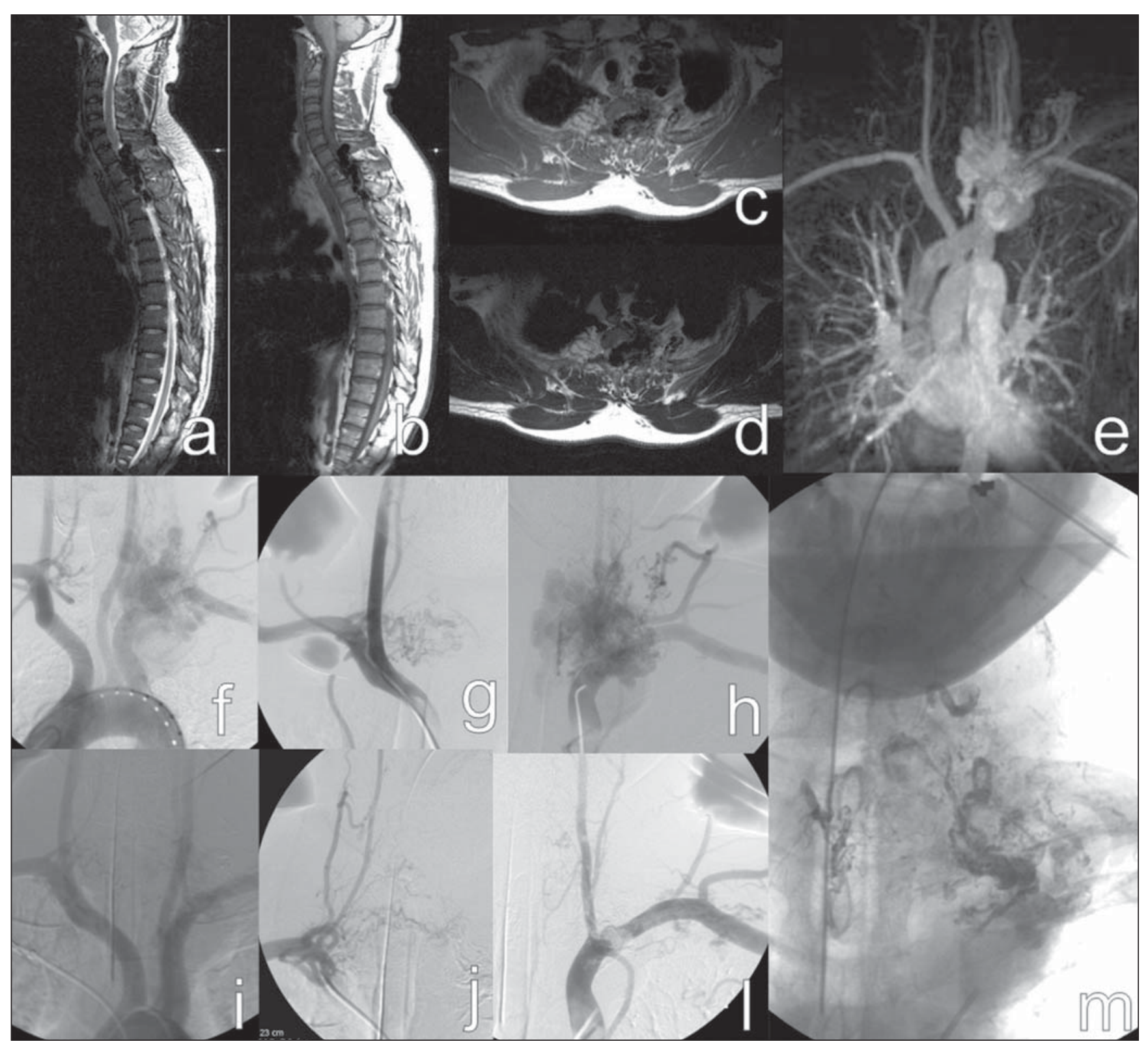

Figura 5. Malformação arteriovenosa. Sexo masculino, 25 anos, tetraplegia de instalação progressiva há dois anos. Ressonância magnética da coluna cervicotorácica, sequências ponderadas em (a) T2 e (b) T1 no plano sagital e ponderadas em (c) T1 e (d) T2 no plano axial, evidencia estrutura serpiginosa cervicotorácica com ausência de sinal em todas as sequências, paravertebral à esquerda, com componente venoso no canal medular, promovendo compressão da medula espinal. (e) Angiorressonância magnética demonstra comunicação de ramos da artéria subclávia esquerda com contrastação precoce da veia subclávia do mesmo lado, interposta por tecido amorfo realçante (nidus). (f,g,h) Angiografia evidencia malformação arteriovenosa suprida por ramos das artérias subclávias. (i,j, I) Angiografia após quatro sessões de embolização por microcateterismo com uso de NBCA evidencia acentuada redução da circulação no nidus. (m) Aspecto do NBCA ocupando a luz dos vasos patológicos ao final da última sessão de embolização. 0 paciente evoluiu com recuperação total das funções motoras.

diagnóstico das MAVs, caracterizada pela contrastação precoce de estruturas venosas e pela presença do nidus malformativo.

\section{Fístulas arteriovenosas (FAVs)}

As FAVs, por sua vez, são diferenciadas das MAVs por apresentarem comunicação direta entre a artéria e a veia. Diferentemente das FAVs intracranianas, as periféricas são geralmente secundárias a processos póstraumáticos ou pós-infecciosos ${ }^{(14)}$. A pele da região afetada geralmente apresenta-se de coloração normal. Pode apresentar frêmito e sopro ao exame físico. A ultrassonografia associada à técnica Doppler demonstra aumento do pico de velocidade sistólico no sistema arterial, que pode se apresentar ectasiado, com veias de drena- gem também ectasiadas e fluxo arterializado. Os achados de ressonância magnética são semelhantes aos das MAVs, exceto pela ausência do nidus malformativo. A arteriografia também é diagnóstica e padrão ouro para o diagnóstico das FAVs, sendo a diferenciação com as MAVs feita pela ausência do nidus malformativo (Figura 7). 


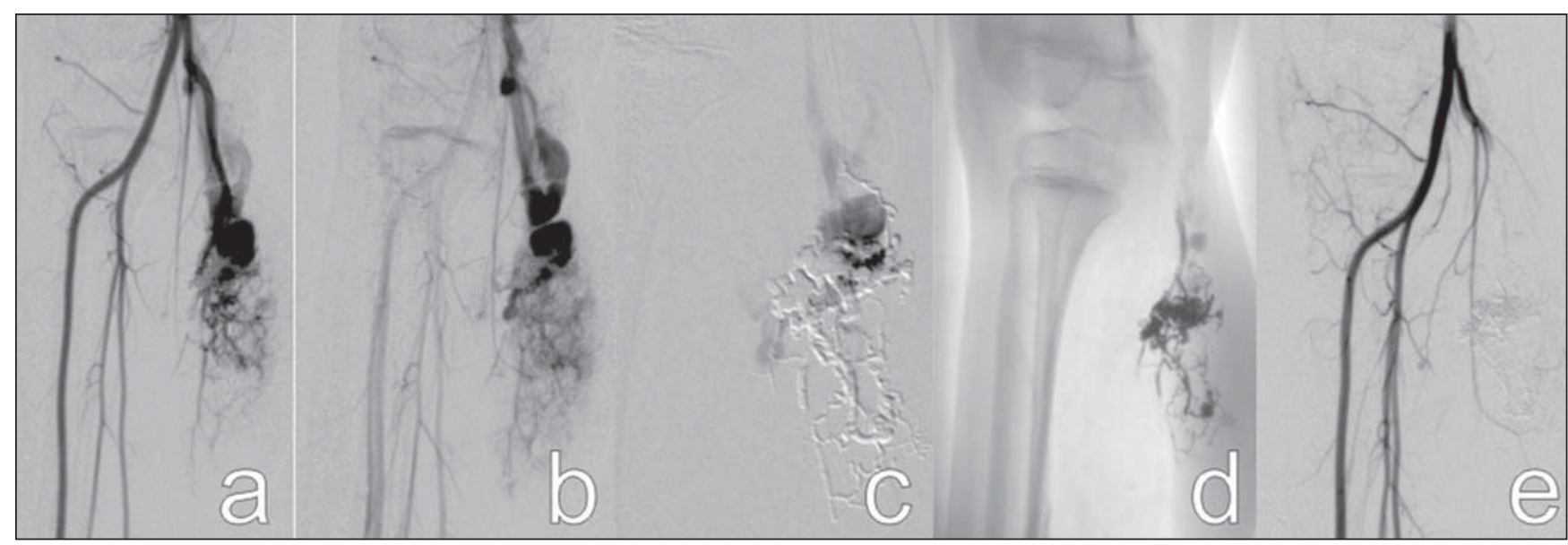

Figura 6. Malformação arteriovenosa. Sexo feminino, 6 anos, abaulamento doloroso na panturrilha direita, com aumento de temperatura e frêmito local. $(\mathbf{a}, \mathbf{b})$ Angiografia seletiva por cateter evidencia malformação arteriovenosa na região posterior da coxa direita. (c) Microcateterismo seletivo realizado durante sessão de embolização com Onyx. (d) Aspecto fluoroscópico do Onyx ocupando o nidus e as artérias nutridoras ao final da sessão. (e) Angiografia seletiva por cateter ao final do procedimento evidencia resolução da malformação arteriovenosa.

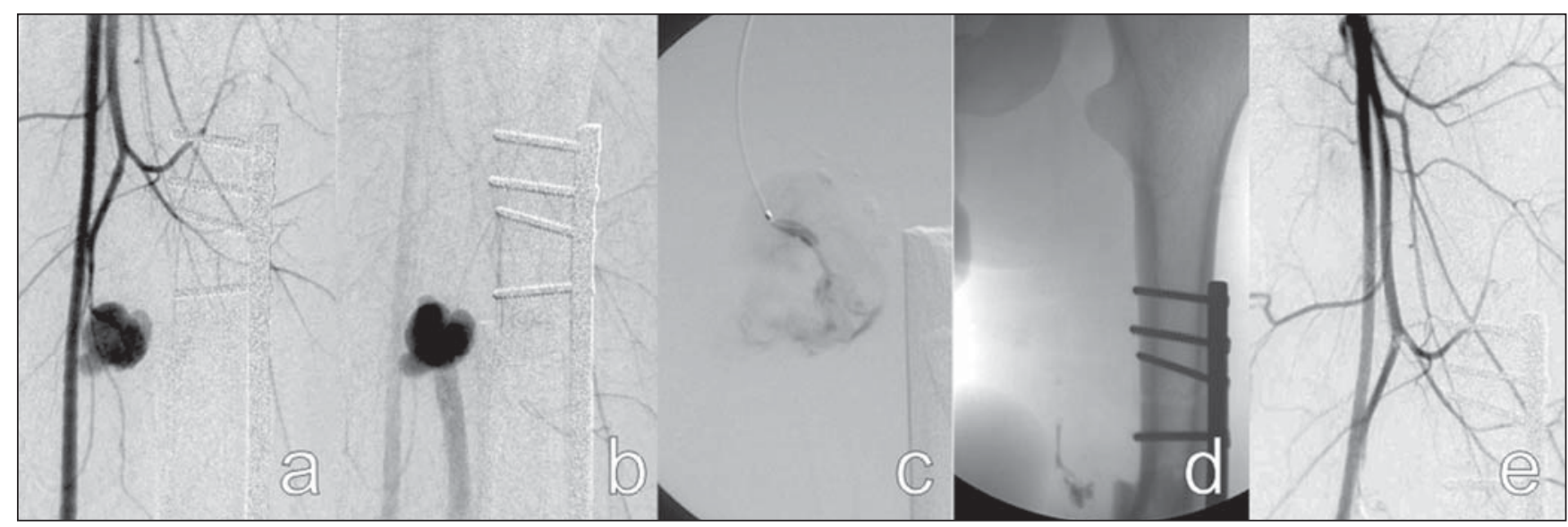

Figura 7. Fístula arteriovenosa. Sexo masculino, 31 anos, acidente automobilístico. Aumento de volume da coxa esquerda após fixação da fratura do fêmur. (a,b) Angiografia seletiva por cateter do membro inferior esquerdo evidenciou fístula arteriovenosa direta entre ramo da artéria femoral profunda e a veia femoral superficial deste lado. (c) A comunicação foi tratada por via percutânea por meio de microcateterismo seletivo e injeção de NBCA. (d) Aspecto fluoroscópico do NBCA ocupando a comunicação arteriovenosa ao final do procedimento. (e) Angiografia seletiva por cateter realizada ao final do procedimento evidencia resolução da comunicação.

\section{Malformações arteriais (MAs)}

As MAs com sintomas clínicos relevantes acometem principalmente a aorta, seus ramos e as artérias dos membros, e englobam os aneurismas, as coarctações e as hipoplasias vasculares. O quadro clínico é variável e depende da localização e do tipo de alteração. A coarctação aórtica é a mais frequente e mais conhecida MA; pode estar acompanhada de malformações cardíacas em aproximadamente $5 \%$ a $8 \%$ dos casos e apresentar-se com hipertensão, insuficiência cardíaca, baixa perfusão dos membros inferiores e cefaleia. Embora seu tratamento, na maioria das vezes, seja cirúr- gico, pode ser realizado por intermédio da radiologia intervencionista, com angioplastia por balão ou stent. Outras MAs incluem a artéria ilíaca interna fetal e a persistência da artéria ciática, que se apresentam com claudicação e isquemia de membros inferiores. A artéria subclávia direita aberrante pode apresentar uma dilatação ou um aneurisma em sua origem (divertículo de Kommerel) e pode apresentar-se assintomática ou com disfagia lusória ${ }^{(\mathbf{1})}$. Podem ser facilmente diagnosticadas com auxílio da angiotomografia computadorizada, angiorressonância magnética e angiografia por cateter.

\section{Malformações complexas}

Abrangem as malformações com dois ou mais tipos histológicos diferentes (capilar-venosa, capilar-linfático-venosa, linfático-venosa, capilar-artério-venosa e capilar-linfático-artério-venosa).

\section{Síndromes associadas a malformações vasculares}

Muitas síndromes apresentam malformações vasculares em sua composição. São exemplos: Sturge-Weber, Klippel-Trenaunay, cutis marmorata teleangiectática congênita, Parkes-Weber, Proteus, Maffucci, entre outras ${ }^{(8)}$. 


\section{OPÇÕES TERAPÊUTICAS E INDICAÇÕES}

O tratamento das malformações vasculares é extremamente variado ${ }^{(\mathbf{5 , 6})}$ e abrange ressecção cirúrgica, terapia a laser ${ }^{(\mathbf{1 5})}$, escleroterapia por punção direita ${ }^{(\mathbf{1 6}, 17)}$ e embolização $\operatorname{arterial}^{(\mathbf{1 8 , 1 9 )}}$, com indicações específicas para cada subgrupo de lesões, com base na sua localização, extensão e classificação. A correta classificação é imperativa para determinar a escolha terapêutica para cada caso, e a escolha errônea de materiais embolizantes pode ser desastrosa. Malformações de baixo fluxo geralmente são tratadas por abordagem percutânea e injeção de agente esclerosante, enquanto para as malformações de alto fluxo o acesso é endovascular com uso de agentes embolizantes permanentes líquidos e sólidos.

O tratamento atualmente indicado para as malformações capilares é o laser corante pulsado (pulsed dye laser - PDL) com comprimento de onda específico para a oxihemoglobina, que promove a coagulação sanguínea no interior da lesão, com clareamento da lesão no decorrer do tratamento ${ }^{(15)}$. Quando o comprimento de onda é determinado para a oxi-hemoglobina, a destruição ocorre quase seletivamente nos vasos sanguíneos patológicos, preservando os tecidos adjacentes, com ausência de cicatriz e ótimos resultados estéticos. Ocasionalmente, a absorção do laser pela melanina epidérmica provoca injúria térmica, sendo necessária associação de resfriamento cutâneo durante a aplicação do laser.

Preconiza-se que o acompanhamento ambulatorial dos pacientes e a decisão terapêutica sejam multidisciplinares, sendo a equipe formada por pediatras, cirurgiões pediátricos, dermatologistas, radiologistas, radiologistas intervencionistas, cirurgiões vasculares ou plásticos, otorrinolaringologistas, oftalmologistas, cirurgiões de cabeça e pescoço, ortopedistas oncológicos, anestesistas, psicólogos, fisioterapeutas e terapeutas ocupacionais $^{(\mathbf{6})}$.

No momento da decisão terapêutica, deve-se levar em conta os riscos do tratamento proposto em relação ao impacto psicológico e morbidades associadas ao curso natural da doença. Indicações absolutas para o tratamento são: hemorragia, insuficiência cardíaca de alto débito, complica- ções secundárias a hipertensão venosa e lesão em região que cause risco à vida, como as vias aéreas. Já as indicações relativas incluem: dor ou desconforto progressivos, alterações funcionais que prejudicam as atividades cotidianas ou a qualidade de vida, deformidade severa, síndrome vascular-óssea (aumento discrepante de um membro), localização da lesão com alto risco de complicações e infecção ou sepse recorrentes ${ }^{(\mathbf{6})}$.

A decisão entre tratamento cirúrgico ou por radiologia intervencionista baseia-se na experiência dos profissionais envolvidos, levando em consideração a opção do paciente ou responsável. Em determinadas situações, pode-se associar os métodos.

\section{Tratamento percutâneo guiado por imagem}

O tratamento percutâneo pode ser realizado por diferentes vias de acesso, com uso de uma gama de agentes embolizantes. A característica determinante para a escolha do método mais adequado é a classificação da lesão quanto à velocidade do fluxo sanguíneo. A embolização transarterial ou transvenosa é preferida para tratamento de malformações de alto fluxo e pode ser realizada com agentes embolizantes sólidos, como as espirais metálicas em platina com destacamento controlado, eventualmente micropartículas de polivinilálcool (PVA), Gelfoam e outros $^{(\mathbf{1 8 - 2 0})}$, ou agentes líquidos adesivos, como o cianoacrilato, e não adesivos, como o Onyx (ev3 Neurovascular, Inc.; Irvine, CA, EUA) ${ }^{(21)}$. Já para o tratamento das lesões de baixo fluxo, opta-se pela escleroterapia percutânea.

A escleroterapia trata-se de punção percutânea direta da lesão com agulha fina, conectada um pequeno extensor, pelo qual é realizada injeção de diferentes substâncias que promovem coagulação sanguínea, desnaturação proteica e um acentuado processo inflamatório local que levará à obliteração das cavidades malformadas. A injeção da maioria dos agentes esclerosantes é feita sob controle fluoroscópico. Punções de lesões profundas podem ser realizadas com auxílio ultrassonográfico ou tomográfico. Diversos agentes esclerosantes já foram usados ${ }^{\mathbf{( 2 2 - 2 4 )}}$, mas o de maior abrangência e de menor custo é o álcool absoluto, misturado a outras substâncias para torná-

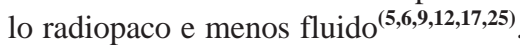

Na embolização transarterial, a lesão é acessada por meio de punção arterial a distância, com uso de cateteres, cateteres-guia e microcateteres, com aplicação de material permanente líquido ou sólido, com intuito de preenchimento dos vasos malformados e resolução das comunicações anômalas. Eventualmente, dependendo da localização da lesão, o acesso também pode

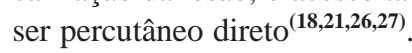

Os procedimentos são realizados sob anestesia geral ou sedação acompanhada de bloqueio anestésico regional, dependendo da localização da lesão. Alguns cuidados pré-operatórios, como avaliação da função renal, avaliação da coagulabilidade sanguínea, contagem de plaquetas, devem ser monitorados e alterações identificadas devem ser corrigidas.

\section{Álcool absoluto}

É o agente mais usado para escleroterapia percutânea, intravenosa ou intra-arterial, dependendo das características hemodinâmicas da lesão. É indicado para malformações de baixo fluxo (Figuras 1, 2 e 4). Pode ser utilizado misturado a contraste iodado ou contraste oleoso à base de óleo de papoula - lipiodol ou Ethiodol (Savage Laboratories; Melville, NY, EUA). Sua ação ocorre por lesão do epitélio do vaso, coagulação sanguínea, trombose e oclusão, com resposta terapêutica em torno de $64 \%$ a $96 \%{ }^{(5,17,28)}$. As complicações do uso do álcool absoluto variam em torno de 7,5\% a 27,95\% e incluem: necrose cutânea, dor transitória, contratura muscular, lesão neurológica (em geral transitória), celulite, trombose venosa profunda, embolia pulmonar, e até choque circulatório ${ }^{(29)}$. A dose máxima considerada segura é de $1 \mathrm{~mL} / \mathrm{kg}$ de peso em cada sessão ${ }^{(\mathbf{1 6})}$; dependendo da extensão e da localização da lesão, múltiplas sessões poderão ser necessárias. Após a realização do procedimento, uma intensa reação inflamatória local é esperada, e se necessário, analgésicos e anti-inflamatórios podem ser utilizados. Estes últimos devem ser evitados ao máximo, pois a atividade inflamatória está associada a melhores respostas terapêuticas.

\section{Bleomicina}

A bleomicina é um antibiótico citotóxico antitumoral que apresenta ação escle- 
rosante quando em contato com o epitélio vascular, efeito colateral que foi utilizado para uso terapêutico pela primeira vez em 1977 para o tratamento de MLs do tipo macrocístico. Sua utilização nas MVPs é mais frequente para as MLs, mas também há relatos de uso para MVs e MAVs ${ }^{(30)}$. Deve ser diluída para uma concentração de $1 \mathrm{mg} / \mathrm{mL}$, aplicada por punção direta na lesão. As complicações mais comuns são celulite, ulceração, queda de cabelo e sintomas semelhantes ao resfriado.

\section{OK-432 (Picibanil) (Chugai Pharmaceutical Co.; Tóquio, Japão)}

É uma solução preparada a partir de células de Streptococcus pyogenes liofilizadas, tratadas com benzilpenicilina. É utilizado para tratamento de MLs, com bons resultados no subtipo macrocístico. É injetado por punção direta da lesão, após aspiração de conteúdo seroso ou esbranquiçado, usualmente sem controle por nenhum método de imagem. Apresenta baixos índices de complicações.

\section{Oleato de etanolamina}

Agente pouco utilizado para o tratamento de MVPs, é empregado mais comumente para varizes esofágicas. Deve ser misturado a contraste iodado não iônico para manter seu efeito surfactante e ser visualizado na fluoroscopia $^{(5)}$. Existem poucos relatos de complicações, porém apresenta pior resposta terapêutica.

\section{Polidocanol}

Mais utilizado para escleroterapia de varizes esofágicas e de membros inferiores. Devido à ação anestésica do polidocanol, não é necessária analgesia durante o procedimento.

\section{N-butil-cianoacrilato (NBCA)}

Agente embolizante líquido adesivo, é utilizado para tratamento de malformações vasculares de alto fluxo, em especial no tratamento das MAVs, por meio de punção direta ou transarterial, com injeção diretamente no nidus malformativo ${ }^{(31)}$ (Figuras 5 e 7). É usado diluído em lipiodol, em concentrações que variam de acordo com a velocidade do fluxo sanguíneo. O lipiodol retarda a polimerização do NBCA e o torna visível à fluoroscopia. O NBCA age poli- merizando-se quando em contato com o sangue, ocupando o espaço vascular de modo permanente, visto que é um agente não absorvível. Necessita experiência do profissional no seu manejo, para reduzir os riscos do procedimento e efeitos colaterais.

\section{Copolímero etileno vinil álcool (Onyx)}

É um polímero biocompatível solúvel em dimetil sulfóxido (DMSO), de ação permanente, não adesivo, que não age por polimerização, mas por precipitação, causando oclusão do vaso onde é injetado. É uma solução composta por copolímero etileno vinil álcool, DMSO e sais de tântalo, que o torna radiopaco. Deve ser mantido em agitação por pelo menos 20 minutos antes do uso, para que os componentes fiquem homogêneos no momento da injeção. Foi utilizado primariamente na embolização de MAVs $^{(32)}$ e FAVs durais intracranianas ${ }^{(33)}$.

Pela sua característica não adesiva, seu tempo de uso é prolongado, com melhores resultados por sessão quando comparado ao NBCA. Pode ser utilizado para tratamento de malformações de alto e baixo fluxos, geralmente por via intra-arterial ou intravenosa ${ }^{(21)}$. Tem papel crescente no tratamento de malformações de alto fluxo em razão do maior controle durante a administração do material, com menores complicações a distância, principalmente nas MAVs (Figura 6).

\section{Espirais metálicas em platina}

Utilizadas primariamente no tratamento de aneurismas cerebrais desde o início dos anos $90^{(\mathbf{3 4})}$, as espirais metálicas em platina com destacamento controlado passaram também a ser usadas no tratamento de outras lesões vasculares cerebrais, como FAVs durais e piais, e periféricas, como nos aneurismas viscerais e malformações vasculares de alto fluxo ${ }^{(35)}$.

São frequentemente utilizadas no tratamento das FAVs, por acesso endovascular por via arterial. Também podem ser utilizadas nas outras malformações vasculares no intuito de reduzir a velocidade do fluxo sanguíneo para permitir o uso mais seguro de agentes esclerosantes.

Suas indicações em MAVs são muito limitadas, pois geralmente causam oclusão proximal dos pedículos, levando a um recrutamento de colaterais para o nidus mal- formativo, o que dificulta uma abordagem posterior e não resulta em nenhuma melhora clínica.

\section{Partículas de polivinilálcool (PVA)}

As partículas de PVA já foram o material de escolha tanto para o tratamento de MAVs periféricas quanto cerebrais. São de fácil manuseio, apresentam-se em diferentes dimensões e são aplicadas por meio de cateterização seletiva ou superseletiva dos vasos nutridores da malformação. Causam redução paliativa do fluxo sanguíneo, mas promovem oclusão proximal e temporária dos vasos, sendo a recorrência dos sintomas muito frequente. Apresentam taxas consideráveis de complicação por embolização de territórios normais e migração para o sistema venoso, podendo causar tromboembolismo pulmonar. Por estes motivos, seu uso para o tratamento das MVs é atualmente muito restrito.

\section{CONCLUSÃO}

O diagnóstico das anomalias vasculares é realizado com base na história clínica e no exame físico. Informações como presença da lesão ao nascimento, crescimento proporcional, período de involução, alterações de pigmentação da pele, efeito de massa, presença de sopro e de síndromes genéticas associadas são de extrema importância. Os exames de imagem, em especial ultrassonografia com Doppler e ressonância magnética, são importantes para avaliar a velocidade de fluxo da lesão, sua real extensão e o envolvimento de estruturas adjacentes. Malformações vasculares de alto fluxo devem ser sempre avaliadas por angiografia para auxiliar na escolha do tratamento. É de extrema importância para o adequado acompanhamento dos pacientes que sejam utilizadas as nomenclaturas corretas aos diferentes subtipos de malformações vasculares, no intuito de evitar iatrogenias terapêuticas. O tratamento percutâneo, seja por punção direita ou endovascular, necessita um treinamento específico da equipe e o conhecimento profundo das técnicas a serem utilizadas.

\section{REFERÊNCIAS}

1. Gloviczki P, Duncan A, Kalra M, et al. Vascular malformations: an update. Perspect Vasc Surg Endovasc Ther. 2009;21:133-48. 
2. Legiehn GM, Heran MK. Venous malformations: classification, development, diagnosis, and interventional radiologic management. Radiol Clin North Am. 2008;46:545-97, vi.

3. Enjolras O, Wassef M, Chapot R. Color atlas of vascular tumors and vascular malformations. New York, NY: Cambridge University Press; 2007.

4. Mulliken JB, Glowacki J. Hemangiomas and vascular malformations in infants and children: a classification based on endothelial characteristics. Plast Reconstr Surg. 1982;69:412-22.

5. Hyodoh H, Hori M, Akiba H, et al. Peripheral vascular malformations: imaging, treatment approaches, and therapeutic issues. Radiographics. 2005;25 Suppl 1:S159-71.

6. Legiehn GM, Heran MK. Classification, diagnosis, and interventional radiologic management of vascular malformations. Orthop Clin North Am. 2006;37:435-74, vii-viii.

7. Moukaddam H, Pollak J, Haims AH. MRI characteristics and classification of peripheral vascular malformations and tumors. Skeletal Radiol. 2009;38:535-47.

8. Garzon MC, Huang JT, Enjolras O, et al. Vascular malformations. Part II: associated syndromes. J Am Acad Dermatol. 2007;56:541-64.

9. Escobar FN, Chamorro FM, Trujillo CIP, et al. Hemangiomas y malformaciones vasculares: enfoque diagnóstico y terapéutico. Rev Colomb Radiol. 2008;19:2409-24.

10. Garzon MC, Huang JT, Enjolras O, et al. Vascular malformations: Part I. J Am Acad Dermatol. 2007;56:353-70; quiz 371-4.

11. Spence J, Krings T, terBrugge KG, et al. Percutaneous sclerotherapy for facial venous malformations: subjective clinical and objective MR imaging follow-up results. AJNR Am J Neuroradiol. 2010;31:955-60.

12. Puig S, Casati B, Staudenherz A, et al. Vascular low-flow malformations in children: current concepts for classification, diagnosis and therapy. Eur J Radiol. 2005;53:35-45.
13. Gontijo B, Pereira LB, Silva CMR. Malformações vasculares. An Bras Dermatol. 2004;79:7-25.

14. González SB, Busquets JC, Figueiras RG, et al. Imaging arteriovenous fistulas. AJR Am J Roentgenol. 2009;193:1425-33.

15. Stier MF, Glick SA, Hirsch RJ. Laser treatment of pediatric vascular lesions: Port wine stains and hemangiomas. J Am Acad Dermatol. 2008;58: 261-85.

16. Puig S, Aref H, Brunelle F. Double-needle sclerotherapy of lymphangiomas and venous angiomas in children: a simple technique to prevent complications. AJR Am J Roentgenol. 2003;180: 1399-401.

17. Górriz Gómez E, Carreira JM. Tratamiento percutáneo de las malformaciones vasculares periféricas con una mezcla de polidocanol y $\mathrm{CO} 2$. experiencia inicial. Radiología. 2008;50:424-9.

18. Nambiar AP, Bozlar U, Angle JF, et al. Initial clinical experience with biopolymer-coated detachable coils (hydrocoil) in peripheral embolization procedures. J Vasc Interv Radiol. 2008;19:9951001.

19. Osuga K, Hori S, Kitayoshi H, et al. Embolization of high flow arteriovenous malformations: experience with use of superabsorbent polymer microspheres. J Vasc Interv Radiol. 2002;13: 1125-33.

20. Kwak BK, Shim HJ. Arterial occlusion using a microguidewire as a radiofrequency electrode. J Endovasc Ther. 2008;15:370-4.

21. Numan F, Omeroglu A, Kara B, et al. Embolization of peripheral vascular malformations with ethylene vinyl alcohol copolymer (Onyx). J Vasc Interv Radiol. 2004;15:939-46.

22. Okazaki T, Iwatani S, Yanai T, et al. Treatment of lymphangioma in children: our experience of 128 cases. J Pediatr Surg. 2007;42:386-9.

23. Emran MA, Dubois J, Laberge L, et al. Alcoholic solution of zein (Ethibloc) sclerotherapy for treatment of lymphangiomas in children. J Pediatr Surg. 2006;41:975-9.
24. Ruiz Jr E, Valera ET, Verissimo F, et al. Uso de OK-432 em crianças com linfangioma. J Pediatr (Rio J). 2004;80:154-8.

25. Rosenblatt M. Endovascular management of venous malformations. Phlebology. 2007;22: 264-75.

26. Tan KT, Simons ME, Rajan DK, et al. Peripheral high-flow arteriovenous vascular malformations: a single-center experience. J Vasc Interv Radiol. 2004; 15:1071-80.

27. Cura M, Elmerhi F, Suri R, et al. Vascular malformations and arteriovenous fistulas of the kidney. Acta Radiol. 2010;51:144-9.

28. Lourenço MA, Gomes CS, Beffa CV, et al. Utilização de álcool absoluto no tratamento das malformações venosas. Radiol Bras. 2001;34:23-7.

29. Lee KB, Kim DI, Oh SK, et al. Incidence of soft tissue injury and neuropathy after embolo/sclerotherapy for congenital vascular malformation. J Vasc Surg. 2008;48:1286-91.

30. Muir T, Kirsten M, Fourie P, et al. Intralesiona bleomycin injection (IBI) treatment for haemangiomas and congenital vascular malformations. Pediatr Surg Int. 2004;19:766-73.

31. Pollak JS, White RI Jr. The use of cyanoacrylate adhesives in peripheral embolization. $\mathrm{J}$ Vasc Interv Radiol. 2001;12:907-13

32. Mounayer C, Hammami N, Piotin M, et al. Nidal embolization of brain arteriovenous malformations using Onyx in 94 patients. AJNR Am J Neuroradiol. 2007;28:518-23.

33. Rezende MT, Piotin M, Mounayer C, et al. Dural arteriovenous fistula of the lesser sphenoid wing region treated with Onyx: technical note. Neuroradiology. 2006;48:130-4.

34. Guglielmi G, Viñuela F. Intracranial aneurysms. Guglielmi electrothrombotic coils. Neurosurg Clin N Am. 1994;5:427-35.

35. Abath C, Andrade G, Cavalcanti D, et al. Complex renal artery aneurysms: liquids or coils? Tech Vasc Interv Radiol. 2007;10:299-307. 